\title{
A Comparative Study of Bioartificial Bone Tissue Poly-L-lactic Acid/Polycaprolactone and PLLA Scaffolds Applied in Bone Regeneration
}

\author{
Weizong Weng, Shaojun Song, Liehu Cao, Xiao Chen, Yuanqi Cai, \\ Haihang Li, Qirong Zhou, Jun Zhang, and Jiacan Su \\ Department of Orthopaedic Surgery, Changhai Hospital, Second Military Medical University, 168 Changhai Road, \\ Shanghai 200433, China
}

Correspondence should be addressed to Jiacan Su; weesonyo@hotmail.com

Received 30 September 2014; Revised 17 December 2014; Accepted 17 December 2014; Published 31 December 2014

Academic Editor: Zhongkui Hong

Copyright (C) 2014 Weizong Weng et al. This is an open access article distributed under the Creative Commons Attribution License, which permits unrestricted use, distribution, and reproduction in any medium, provided the original work is properly cited.

\begin{abstract}
Bioartificial bone tissue engineering is an increasingly popular technique to repair bone defect caused by injury or disease. This study aimed to investigate the feasibility of PLLA/PCL (poly-L-lactic acid/polycaprolactone) by a comparison study of PLLA/PCL and PLLA scaffolds applied in bone regeneration. Thirty healthy mature New Zealand rabbits on which $15 \mathrm{~mm}$ distal ulna defect model had been established were selected and then were divided into three groups randomly: group A (repaired with PLLA scaffold), group B (repaired with PLLA/PCL scaffold), and group C (no scaffold) to evaluate the bone-remodeling ability of the implants. Micro-CT examination revealed the prime bone regeneration ability of group B in three groups. Bone mineral density of surgical site in group B was higher than group A but lower than group C. Meanwhile, the bone regeneration in both groups A and B proceeded with signs of inflammation for the initial fast degradation of scaffolds. As a whole, PLLA/PCL scaffolds in vivo initially degrade fast and were better suited to repair bone defect than PLLA in New Zealand rabbits. Furthermore, for the low mineral density of new bone and rapid degradation of the scaffolds, more researches were necessary to optimize the composite for bone regeneration.
\end{abstract}

\section{Introduction}

Bone transplantation can heal bone defect effectively which is a difficulty in orthopaedic treatment caused by trauma, chronic infection, and bone tumours [1-3]. Traditional bone defect repair procedures utilize autogenous bone as a graft to provide the osteoinductive and osteoconductive components necessary for the formation of new bone at the surgical site; it has long been the gold standard for orthopedics. Complications associated with the harvest of autograft, such as limited quantities, donor-site morbidity, blood loss, and increased operative time, have prompted the search for the suitable alternatives [4]. Artificial bones, such as hydroxyapatite, calcium acid phosphate, and corals, have similar structure, good biocompatibility, biodegradability, and conductivity ability relative to human bone. Thus, bioartificial bone tissue engineering becomes an increasingly popular technique to repair bone defect. Previous work has led to the development of a bone-like nanohydroxyapatite/collagen (nHAC) composite; however, its mechanical properties were too weak for practical application [4]. PLLA (poly-L-lactic acid) was applied in surgical suture, implant, and drug delivery system approved by FDA $[5,6]$ for its good biocompatibility, biodegradability ability, and high mechanical strength. However, PLLA has its limitation in bioartificial bone tissue engineering research for its physicochemical properties such as weak resilience [7] and hydrolysate lactate which misfit the weak alkaline environment of human body. Thus, applying composite restoration materials to make up the disadvantages of PLLA becomes a research hotspot currently. PCL (polycaprolactone) possessed good biocompatibility, high permeability to small drug molecule, good resilience, slow degradation, and no acidic degradation products, which can obviate the disadvantages of PLLA [8-10]. For the good tenacity of PCL and hydrophilicity 
of PEG (polyethylene glycol), Jiang et al. [11] composed PLLA/PCL/PEG blend fiber membranes using electrostatic discharge technology to strengthen cellulose's hydrophilicity and tenacity. Sarazin et al. [12] prepared PLLA/PCL macroporous scaffold with high mechanical strength, $1.5 \mathrm{~mm}$ bore diameter, large surface area, good hydrophilicity, and $25 \%-$ $38 \%$ crystallinity. For the characteristics such as hydrophily, degradable and low antigenicity of gelatin and hypocrystalline, and high tenacity of PCL, Gungor-Ozkerim et al. [13] prepared gelatin/polylactic acid/polycaprolactone composite material with high hydrophilicity and tenacity to promote cell adherence and differentiation, yet out of in vivo study. The environment in vivo, including the ease of substance diffusion and the existence of enzymes, is usually regarded as accelerating the degradation of the polymer scaffold. Therefore, to optimize the materials for tissue engineering, experiments in vivo to determine the relationship between the scaffold disappearance and the new tissue restoration are necessary [14]. Walton and Cotton reported a 3-year in vivo study to evaluate histologically the fate of PLLA implanted into the bone of sheep, in which certain limitations identified were suggested as the slow degradation rate of PLLA [15]. It is said that the copolymers with these components degrade faster than either homopolymer alone [16]. Sadiasa et al. performed a study focused on in vitro and 2-month in vivo evaluation of porous PCL-PLLA 3D polymer scaffolds for bone tissue engineering applications in rabbits. In that study, a bone defect with a diameter and depth of $5 \mathrm{~mm}$ was produced, and the defect was completely healed at 8 weeks after surgery [17]. Critical size defect (CSD) means the critical size of bone defect for animals to regeneration or less than $10 \%$ regeneration rate in their life cycle [18], which is generally identified as 1.5-2.5 times the bone diameter [7]. A good animal bone defect model, which can eliminate the self-repair ability of bone, is crucial and beneficial to bone defect research. Therefore, in this study, biocompatibility, degradability, regenerate time, regenerate degree, and new bone mineral density in New Zealand rabbits after PLLA and PLLA/PCL scaffolds were implanted into defect site were compared, which aimed to investigate the applications of PLLA/PCL in vivo for bone regeneration for large defects.

\section{Material and Methods}

2.1. Preparation of PLLA and PLLA/PCL Scaffolds. Thermally induced phase separation (TIPS) process was adopted to prepare PLLA and PLLA/PCL scaffolds. The preparations are as follows: a certain quality of PLLA was made into liquor by $10 \%(\mathrm{w} / \mathrm{v})$ tetrahydrofuran at $60^{\circ} \mathrm{C}$ and then was kept in temperature at $-80^{\circ} \mathrm{C}$ overnight after the liquor was injected into mold, and then the samples were soaked in deionized water at $0^{\circ} \mathrm{C}$ for two days, followed by freeze-drying. The preparations of PLLA/PCL macroporous scaffold are similar to PLLA, mixed PLLA, and PCL with a proportion of $1: 100$.

2.2. Surgery In Vivo New Zealand Rabbits. Thirty healthy mature New Zealand rabbits (2.0-2.5 kilograms) were provided by the Second Military Medical University Animal Laboratory and then were divided into 3 groups randomly
(10 rabbits each group): group A (PLLA dense scaffold group), group B (PLLA/PCL macroporous scaffold group), and group $\mathrm{C}$ (no scaffold as control group). The circumference of ulna defects of 5 randomly selected New Zealand rabbits was measured before the surgery. A large segmental defect with $1.5 \mathrm{~cm}$ length $1 \mathrm{~cm}$ near the ulnar styloid on New Zealand rabbit's right upper limb was produced after anesthetization. PLLA dense scaffold and PLLA/PCL macroporous scaffold with $15 \mathrm{~mm}$ height and $6 \mathrm{~mm}$ diameter were implanted in the defect site in groups A and B, respectively, and no scaffold was implanted in control group. Penicillin sodium ( 0.8 million u) was injected daily for 7 days after surgery to prevent infection.

\subsection{Postoperative Body Temperature, Swelling, and Exudation} Situation. After surgery, temperatures of $30 \mathrm{New}$ Zealand rabbits were detected weekly by using electric thermometer for 5 weeks; then the results were analyzed by One-Way ANOVA. After surgery, swelling and exudation situation was observed and circumference of midbone defect was measured on the last day of each week for 5 weeks.

2.4. X-Ray Radiation Examination. To ensure the success of modeling and check out the difference between individuals, $\mathrm{X}$-ray radiation examination was conducted immediately after surgery. Radioactivity examination was also performed monthly for 3 months to observe porosis and degradation of scaffolds.

2.5. Postoperative Biopsy. One rabbit randomly selected from each group was sacrificed for monthly tissue sampling in the first 3 months after surgery. Bone regeneration, neutrophilia, and degradation of scaffolds were detected by using hematoxylin and eosin (HE) staining technology.

2.6. Micro-CT Examination. After three months of implantation, 5 rabbits in 3 groups (2 from group A, 2 from group $\mathrm{B}$, and 1 from group $\mathrm{C}$ ) were selected randomly for tissue sampling and then the mineral density of new bone and mass loss which indicated the degradation rate of scaffolds were detected with micro-CT examination. Also mass loss $(\%)=$ (initial scaffold volume - remaining scaffold volume)/initial scaffold volume $\times 100 \%$.

2.7. Data Analysis. $t$-test, chi-square test, and repeated measurement of multifactors statistical method in SPSS21 were used for data analysis. A value of $P<0.05$ was considered to be statistically significant. During the experiment, the scaffold in a rabbit of group B was discharged from body, so this rabbit was not counted for data analysis.

\section{Results}

3.1. Postoperative Body Temperature, Swelling, and Exudation Situation. The New Zealand rabbits were kept on normal diet for 4 days after surgery. Temperatures of each rabbit were detected weekly with electric thermometer before and 5 weeks after surgery (Figure 1). The data were analysed by OneWay ANOVA. Two weeks after surgery, significant difference 


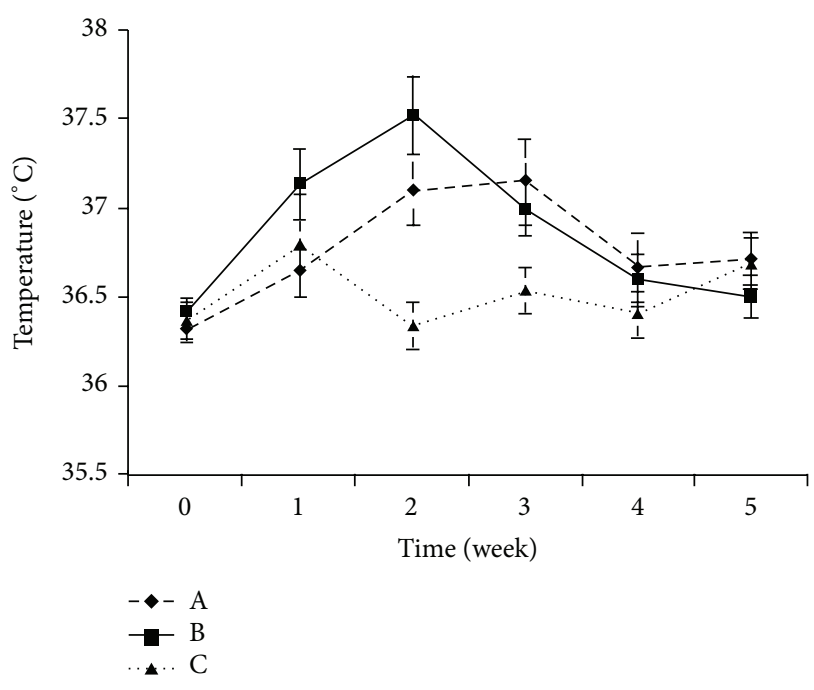

FIGURE 1: Temperature in each group.

TABLE 1: Swelling situation at surgical site.

\begin{tabular}{cccccc}
\hline & $1 \mathrm{w}$ & $2 \mathrm{w}$ & $3 \mathrm{w}$ & $4 \mathrm{w}$ & $5 \mathrm{w}$ \\
\hline $\mathrm{A}$ & + & ++ & +++ & ++ & + \\
$\mathrm{B}$ & ++ & +++ & + & - & - \\
$\mathrm{C}$ & - & - & - & - & - \\
\hline
\end{tabular}

Note: “-" means no swelling $(<0.5 \mathrm{~cm})$; “+” means less swelling $(0.5-$ $1 \mathrm{~cm})$; “++" means medium swelling $(1-2 \mathrm{~cm})$; “+++” means severe swelling $(>2 \mathrm{~cm})$.

TABLE 2: The exudation situation at surgical site.

\begin{tabular}{cccccc}
\hline & $1 \mathrm{w}$ & $2 \mathrm{w}$ & $3 \mathrm{w}$ & $4 \mathrm{w}$ & $5 \mathrm{w}$ \\
\hline $\mathrm{A}$ & - & + & ++ & - & - \\
$\mathrm{B}$ & + & ++ & - & - & - \\
$\mathrm{C}$ & - & - & - & - & - \\
\hline
\end{tabular}

Note: “-” means no exudation; “+” means little exudation; “++" means lots of exudation.

in temperature was detected among three groups $(P<0.01)$, including significant difference between group $B$ and group $\mathrm{C}(P=0.000)$, group A and group $\mathrm{C}(P=0.007)$, while there was no significant difference between group $\mathrm{A}$ and group $\mathrm{B}$ $(P=0.124)$.

No swelling or exudation was observed in group C within 5 weeks, and the damaged skin was healed on 14th day after surgery. As shown in Tables 1 and 2, PLLA density scaffold degraded fast in the first 3 weeks and then progressively slower. PLLA/PCL macroporous scaffold in group B degraded faster than group A and possessed similar degradation rule: with rapid initial degradation rate and then progressively slower rate. Similar swelling situation was detected between group A and group B $(P=0.822)$, while group $\mathrm{C}$ showed significant difference to groups $\mathrm{A}$ and $\mathrm{B}$ $(P<0.01)$.

3.2. X-Ray Radiation Examination. X-ray radiation examination was conducted on right upper limb of 30 New Zealand rabbits immediately after surgery. Sufficient amount of scaffolds had been implanted into the bone defect site and the diameter of scaffold was bigger than ulna, indicating that it is a successful modeling for New Zealand rabbits bone defect. Small callus tissue and remaining scaffold can be observed in group A and group B 1 month after implantation; at the same time, periosteal proliferation was found in samples in group C. There was no statistically significant difference among three groups $(P=0.851)$ according to chi-square test. At 2 months, plenty of calluses were expansively grown in group B; big callus volume was formed at the terminals of defect site in group A; no callus was formed in 8 samples of group $\mathrm{C}$, and the difference among three groups was statistically significant $(P<0.01)$ according to chi-square test. After 3 months, the volume of callus in group B was bigger than group $\mathrm{A}$, and group $\mathrm{C}$ had marrow cavity sclerosis and bone atrophy which was typical bone nonunion performance. The difference among three groups was also significant $(P<$ 0.01 ). The effective rate in group A was $14.29 \%$ (1 of 7 ulnas was healed), while the effective rate of group B was $33.33 \%$ (2 in 6). We excluded 2 dead rabbits in each group which was selected for histology HE staining test in the first 2 months. Figure 2 shows the example radiographs obtained at the $3 \mathrm{rd}$ month after surgery in three groups.

3.3. Histological Examination. As shown in Figure 3, osteoblasts and neutrophil were observed in group A and group B, suggesting inflammatory response to the implantation. Remaining scaffold materials were observed in both group A and group B at 3 months. Moreover, there were no osteoblasts formed within 3 months after surgery in group C.

3.4. Micro-CT Examination. Micro-CT examination of 5 rabbits (2 from group A, 2 from group B, and 1 from group C) was conducted 3 months after surgery. The shapes of the rabbit bones at 3 months were shown in Figure 4 . The bone defect in group A was not completely repaired, which in group B was completely repaired, while group C showed typical bone nonunion. The number of trabeculae per $\mathrm{mm}$ in groups $\mathrm{A}, \mathrm{B}$, and $\mathrm{C}$ was $0.40,0.17$, and 0.34 , respectively. The bone mineral density in groups $\mathrm{A}, \mathrm{B}$, and $\mathrm{C}$ was $1.25434 \mathrm{mg} / \mathrm{cc}, 1.32521 \mathrm{mg} / \mathrm{cc}$, and $1.3349 \mathrm{mg} / \mathrm{cc}$, respectively, and the high bone density in group $\mathrm{C}$ resulted from osteosclerosis. The mass losses of PLLA and PLLA/PCL were $63.575 \%$ and $67.195 \%$, respectively. These two scaffolds have fast initial degradation rate and then slowed down and cannot completely degrade 3 months after implantation.

\section{Discussion}

A desired animal bone defect model is crucial and beneficial to bone defect research. In our study, the measured circumference was $4.3640 \pm 0.3464 \mathrm{~mm}$, indicating the $15 \mathrm{~mm}$ distal ulna defect as a suitable bone defect model.

In our study, these two kinds of scaffolds have fast initial degradation rate: the mass loss of PLLA was 25.2\% on sixteenth day but $42.8 \%$ of PLLA/PCL, which was 1.7 


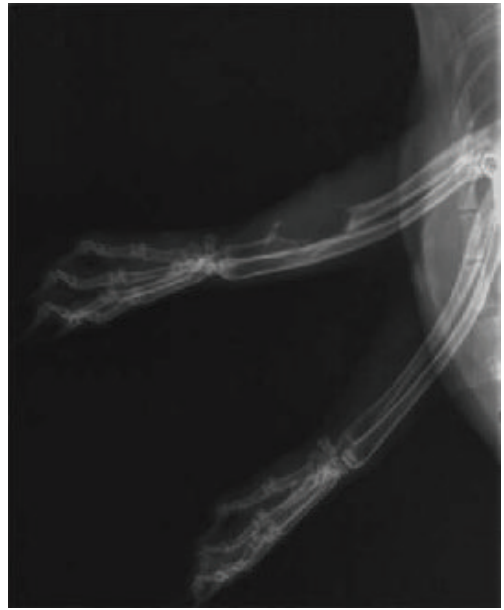

(a)

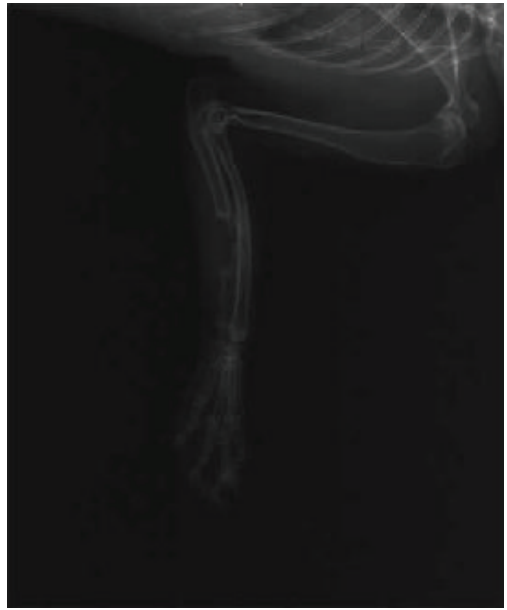

(b)

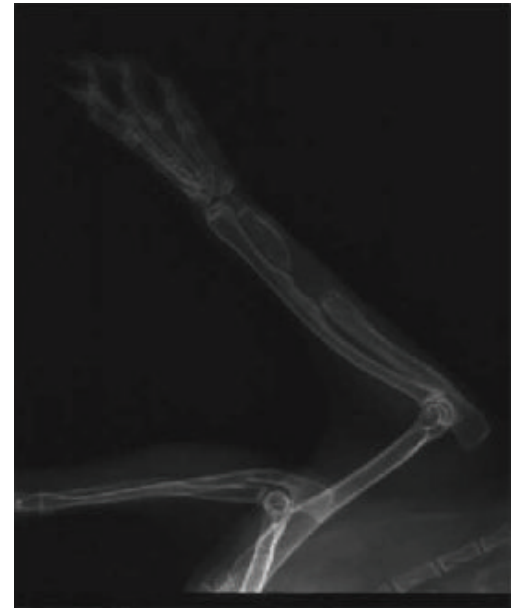

(c)

FIGURE 2: Radiographs obtained at the 3rd month after surgery in groups A (a), B (b), and C (c).

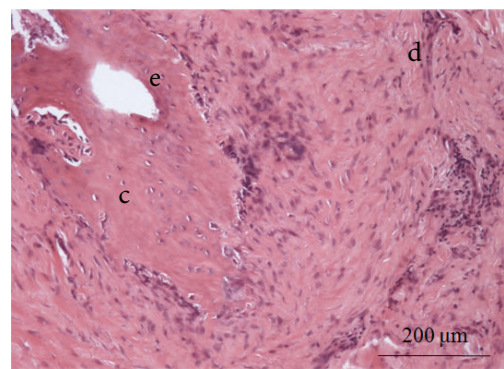

(a)

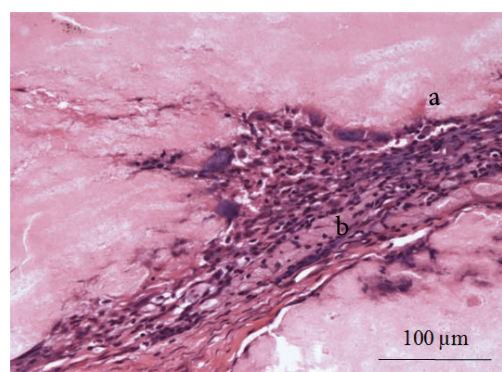

(d)

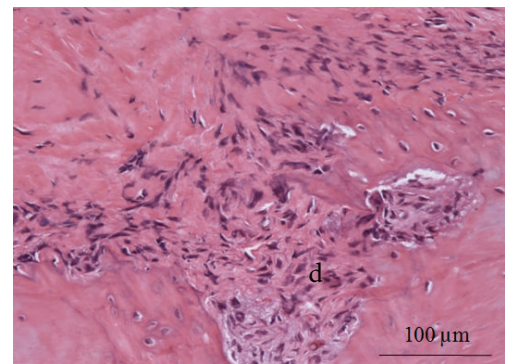

(g)

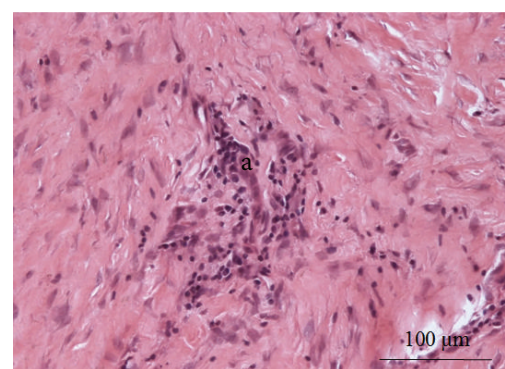

(b)

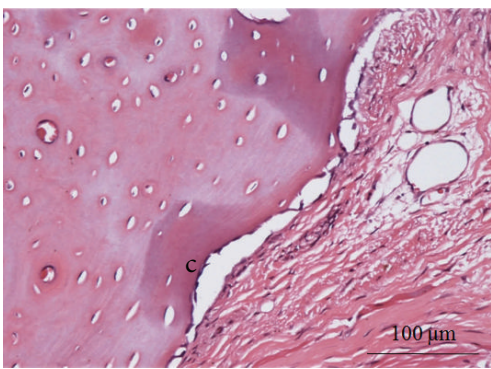

(e)

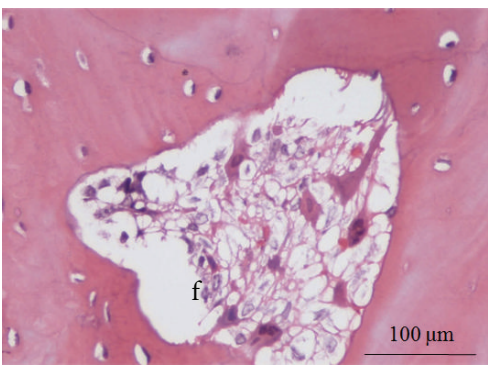

(h)

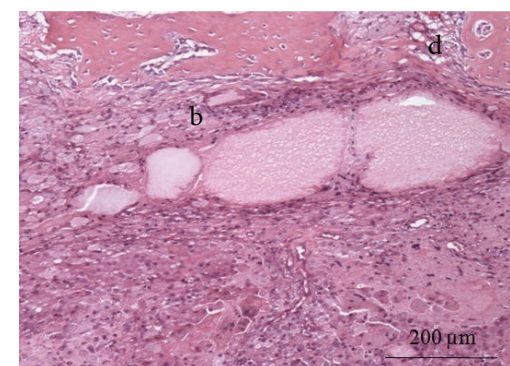

(c)

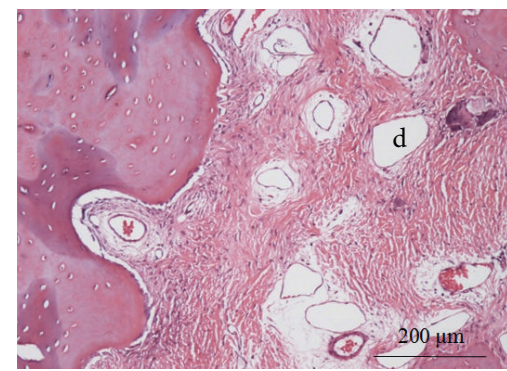

(f)

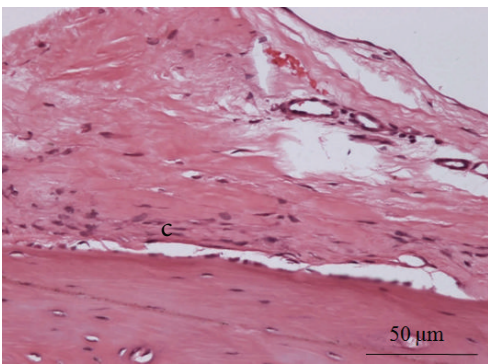

(i)

Figure 3: Histology of surgical site in groups A ((a), (b), and (c)), B ((d), (e), and (f)), and C ((g), (h), and (i)) 3 months after implantation. The alphabet in the figures indicated the following: a: inflammatory cells; b: scaffold materials; c: osteoblast; $d$ : marrow cavity; e: trabecula; f: osteoclast. 


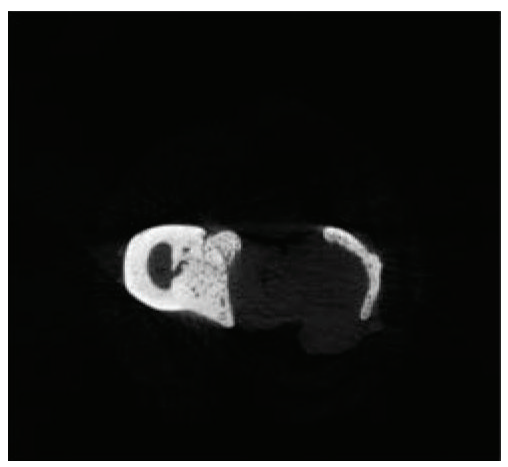

(a)

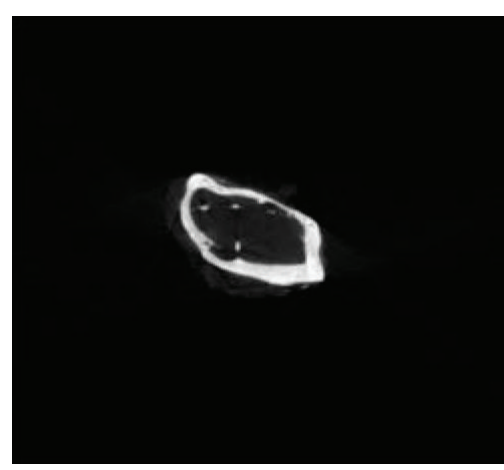

(d)

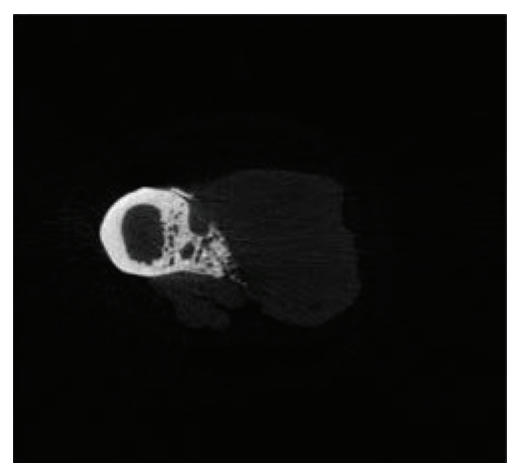

$(\mathrm{g})$

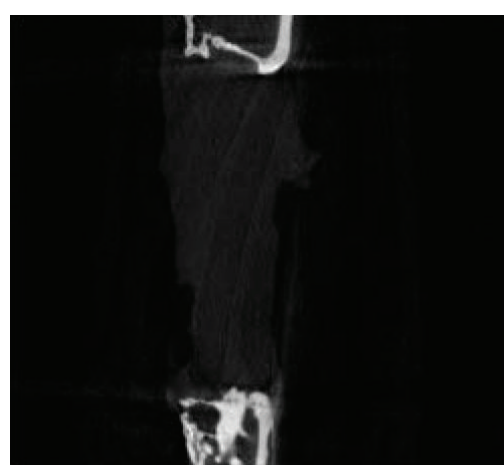

(b)

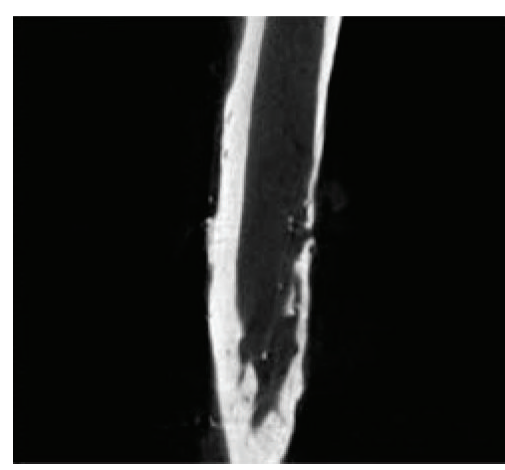

(e)

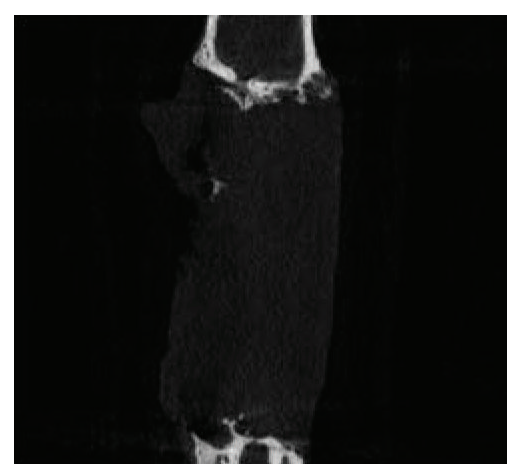

(h)

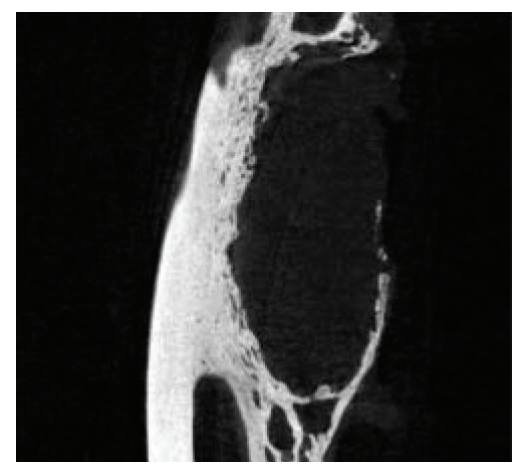

(c)

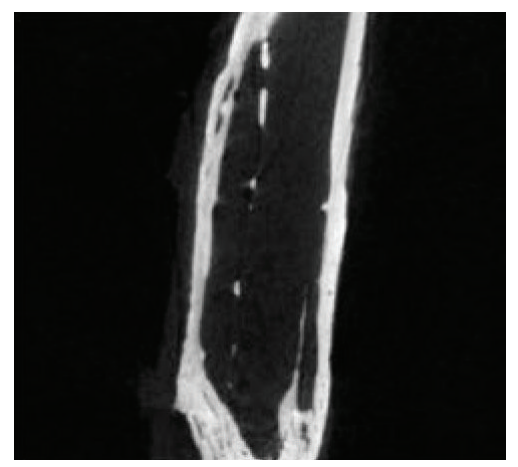

(f)
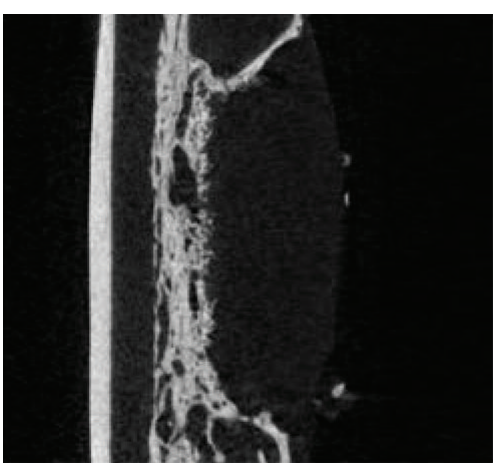

(i)

FIGURE 4: Micro-CT images show the transverse section (left), vertical plane (middle), and coronal plane (right) of defect site in group A ((a), (b), and (c)), group B ((d), (e), and (f)), and group C ((g), (h), and (i)) 3 months after surgery.

times PLLA. Three months after surgery, the mass losses of PLLA and PLLA/PCL were increased to $63.57 \%$ and $67.195 \%$, respectively. The increased temperature in this study was consistent with degradation rate of the implants but unrelated to the infection caused by implantation. Swelling, exudation which indicated inflammatory reaction, and dissatisfied compatibility were observed in the first 3 weeks of group A and group B. During the 5 weeks' detection, there is no significant difference on swelling situation between groups A and $\mathrm{B}(P=0.822)$, while group $\mathrm{C}$ has significant difference to those in groups $\mathrm{A}$ and $\mathrm{B}(P<0.01)$, as we all know that it will bring side effect on bone regeneration when degradation rate is excessively fast or slow. In the study of Sadiasa et al., a defect with a diameter and depth of $5 \mathrm{~mm}$ was produced and scaffolds with PLLA/PCL ratio of $90: 10$ and $70: 30$ were implanted into the defect site. Eight weeks after the surgery, the wounds were completely healed without any inflammation or infection occurring in the rabbits. As a result of their study, scaffolds with PLLA/PCL ratio of $90: 10$ degraded faster than scaffolds with PLLA/PCL ratio of $70: 30$ [17]. Even though in that study a relative small defect was produced when compared to ours, the conclusion also enlightens us that we can control the degradation rate of scaffolds by design of the optimal PLLA/PCL ratio. Hence, our future study will focus on improving the PLLA and PLLA/PCL scaffolds by enhancing their hydrophobicity and controlling the degradation rate, which have been reported frequently in recent works $[13,19]$.

The results of $\mathrm{X}$-ray radiation examination indicated that bone regeneration was initially formed and then stopped 
because of the limited bone self-repair ability. Significant difference appeared at 3 months $(P<0.01)$ : the volume of callus was group $\mathrm{B}>$ group $\mathrm{A}>$ group $\mathrm{C}$. Marrow cavity sclerosis and bone atrophy emerged in group $\mathrm{C}$, indicating typical bone nonunion. These results revealed PLLA and PLLA/PCL as promising scaffold carrier materials for bone defect repairing research.

Both groups $\mathrm{A}$ and $\mathrm{B}$ can achieve the purpose of repairing bone defect in 3 months. The effective rate of group A and group B was $14.29 \%$ and $33.33 \%$, respectively, which was significantly different. The bone mineral density of group $\mathrm{A}$ is also lower than group B. Considering all of these results, we conclude that PLLA and PLLA/PCL scaffolds have slow long-term degradation rate and low bone mineral density because of their bone conductibility ability but lack of osteoinductibility. Previous studies demonstrated that osteoinductibility could be enhanced by combining growth factors and seed cells with scaffold composite material [2022]. For instance, Li proved P24/nHAC/PLLA is better than nHAC/PLLA and rhBMP-2/nHAC/PLLA in bone defect study [23]. Therefore, we can improve the hydrophobicity of the polymer by producing scaffolds composed of PLLA/PCL and specific growth factors in our future study.

\section{Conclusion}

The $15 \mathrm{~mm}$ ulna bone defects model of New Zealand white rabbits can eliminate the bone self-repair ability and eventually formed bone nonunion, which is indicated as a satisfactory bone defect model. The initial degradation rates of PLLA and PLLA/PCL scaffolds were rapid, among which PLLA/PCL degraded faster than PLLA. Moreover, both PLLA and PLLA/PCL scaffolds possessed dissatisfactory histocompatibility and could cause mild inflammatory reaction. At 3 months, as the bone defect in group B was partly repaired, remaining PLLA and PLLA/PCL scaffolds were detected at surgical site in New Zealand white rabbits, indicating the long-term degradation rate of scaffolds was slower than the rate of tissue formation. PLLA and PLLA/PCL scaffolds have slow degradation rate and low bone mineral density because of their lack of osteoinductibility. The bone mineral density and callus formation of PLLA/PCL are better than PLLA which revealed that the PLLA/PCL was more suitable for bone regeneration. In the future study, we aim to improve the degradability, osteogenic ability, and cell adhesion of PLLA/PCL scaffold to make it a more desirable composite material for bone regeneration.

\section{Conflict of Interests}

The authors have declared that no competing interests exist.

\section{Authors' Contribution}

Weizong Weng and Shaojun Song contributed to the work equally and should be regarded as co-first authors.

\section{Acknowledgment}

This work was supported by the National Natural Science Foundation of China (31271031).

\section{References}

[1] G. S. Kalra, P. Goel, and P. K. Singh, "Reconstruction of posttraumatic long bone defect with vascularised free fibula: a series of 28 cases," Indian Journal of Plastic Surgery, vol. 46, no. 3, pp. 543-548, 2013.

[2] T. Liu, X. Zhang, Z. Li, and D. Peng, "Management of combined bone defect and limb-length discrepancy after tibial chronic osteomyelitis," Orthopedics, vol. 34, no. 8, pp. e363-e367, 2011.

[3] C. Gebert, M. Wessling, G. Gosheger et al., "Pelvic reconstruction with compound osteosynthesis following hemipelvectomy: a clinical study," Bone and Joint Journal, vol. 95, no. 10, pp. 14101416, 2013.

[4] S. S. Liao, F. Z. Cui, W. Zhang, and Q. L. Feng, "Hierarchically biomimetic bone scaffold materials: nano-HA/collagen/PLA composite," Journal of Biomedical Materials Research, vol. 69, no. 2, pp. 158-165, 2004.

[5] R. Cancedda, P. Giannoni, and M. Mastrogiacomo, "A tissue engineering approach to bone repair in large animal models and in clinical practice," Biomaterials, vol. 28, no. 29, pp. 4240-4250, 2007.

[6] J. C. Reichert, S. Saifzadeh, M. E. Wullschleger et al., "The challenge of establishing preclinical models for segmental bone defect research," Biomaterials, vol. 30, no. 12, pp. 2149-2163, 2009.

[7] E. A. Horner, J. Kirkham, D. Wood et al., "Long bone defect models for tissue engineering applications: criteria for choice," Tissue Engineering Part B, Reviews, vol. 16, no. 2, pp. 263-271, 2010.

[8] Y. Cha and C. G. Pitt, "The biodegradability of polyester blends," Biomaterials, vol. 11, no. 2, pp. 108-112, 1990.

[9] M.-A. Benoit, B. Baras, and J. Gillard, "Preparation and characterization of protein-loaded poly( $\varepsilon$-caprolactone) microparticles for oral vaccine delivery," International Journal of Pharmaceutics, vol. 184, no. 1, pp. 73-84, 1999.

[10] D.-Y. Lee, B.-H. Choi, J.-H. Park et al., "Nerve regeneration with the use of a poly(L-lactide-co-glycolic acid)-coated collagen tube filled with collagen gel," Journal of Cranio-Maxillofacial Surgery, vol. 34, no. 1, pp. 50-56, 2006.

[11] K. F. Jiang, G. Y. Liao, H. Xia, and X. L. Xie, "Structure and properties of electrospun PLLA/PCL/PEG blend fibrous membranes," Journal of Functional Materials, vol. 9, pp. 15691572, 2011.

[12] P. Sarazin, X. Roy, and B. D. Favis, "Controlled preparation and properties of porous poly(L-lactide) obtained from a cocontinuous blend of two biodegradable polymers," Biomaterials, vol. 25, no. 28, pp. 5965-5978, 2004.

[13] P. S. Gungor-Ozkerim, T. Balkan, G. T. Kose, A. S. Sarac, and F. N. Kok, "Incorporation of growth factor loaded microspheres into polymeric electrospun nanofibers for tissue engineering applications," Journal of Biomedical Materials Research Part A, vol. 102, no. 6, pp. 1897-1908, 2014.

[14] Y. H. Gong, Q. L. Zhou, C. Y. Gao, and J. C. Shen, "In vitro and in vivo degradability and cytocompatibility of poly(l-lactic acid) scaffold fabricated by a gelatin particle leaching method," Acta Biomaterialia, vol. 3, no. 4, pp. 531-540, 2007. 
[15] M. Walton and N. J. Cotton, "Long-term in vivo degradation of poly-L-lactide (PLLA) in bone," Journal of Biomaterials Applications, vol. 21, no. 4, pp. 395-411, 2007.

[16] J. Holmbom, A. Södergård, E. Ekholm et al., "Long-term evaluation of porous poly( $\varepsilon$-caprolactone-co-L-lactide) as a bone-filling material," Journal of Biomedical Materials Research Part A, vol. 75, no. 2, pp. 308-315, 2005.

[17] A. Sadiasa, T. H. Nguyen, and B. T. Lee, "In vitro and in vivo evaluation of porous PCL-PLLA 3D polymer scaffolds fabricated via salt leaching method for bone tissue engineering applications," Journal of Biomaterials Science, vol. 25, no. 2, pp. 150-167, 2014.

[18] A. Berner, J. C. Reichert, M. B. Müller et al., "Treatment of long bone defects and non-unions: from research to clinical practice," Cell and Tissue Research, vol. 347, no. 3, pp. 501-519, 2012.

[19] X. Chen, A. H. Schmidt, D. T. Tsukayama, C. A. Bourgeault, and W. D. Lew, "Recombinant human osteogenic protein-1 induces bone formation in a chronically infected, internally stabilized segmental defect in the rat femur," Journal of Bone and Joint Surgery-Series A, vol. 88, no. 7, pp. 1510-1523, 2006.

[20] S. Shirasawa, I. Sekiya, Y. Sakaguchi, K. Yagishita, S. Ichinose, and T. Muneta, "In vitro chondrogenesis of human synoviumderived mesenchymal stem cells: optimal condition and comparison with bone marrow-derived cells," Journal of Cellular Biochemistry, vol. 97, no. 1, pp. 84-97, 2006.

[21] M. Y. Krasko, J. Golenser, A. Nyska, M. Nyska, Y. S. Brin, and A. J. Domb, "Gentamicin extended release from an injectable polymeric implant," Journal of Controlled Release, vol. 117, no. 1, pp. 90-96, 2007.

[22] K. L. Garvin, J. A. Miyano, D. Robinson, D. Giger, J. Novak, and S. Radio, "Polylactide/polyglycolide antibiotic implants in the treatment of osteomyelitis," Journal of Bone and Joint SurgerySeries A, vol. 76, no. 10, pp. 1500-1506, 1994.

[23] J. F. Li, J. J. Hong, Q. X. Zheng et al., "Repair of rat cranial bone defects with nHAC/PLLA and BMP-2-related peptide or rhBMP-2," Journal of Orthopaedic Research, vol. 29, no. 11, pp. 1745-1752, 2011. 

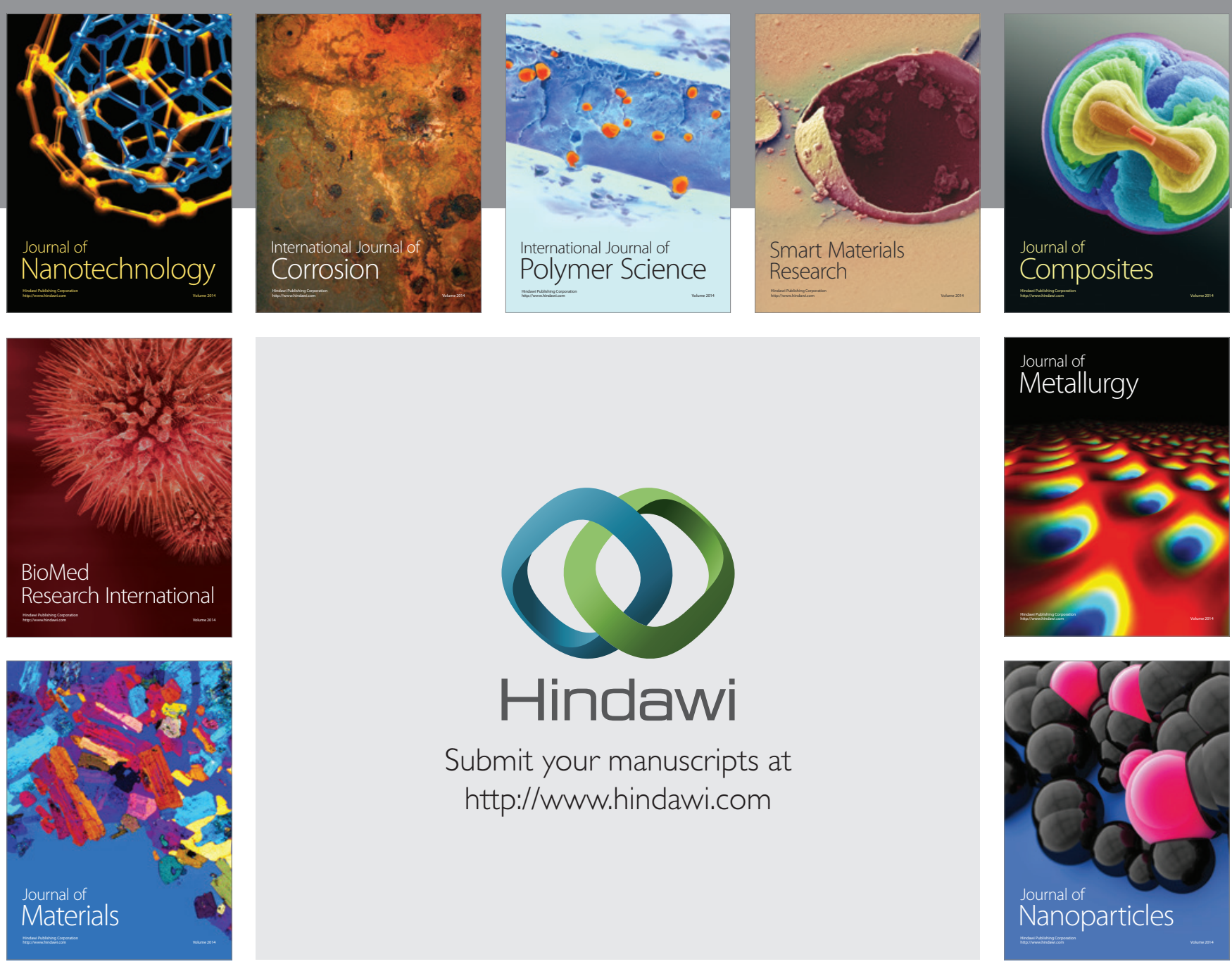

Submit your manuscripts at http://www.hindawi.com
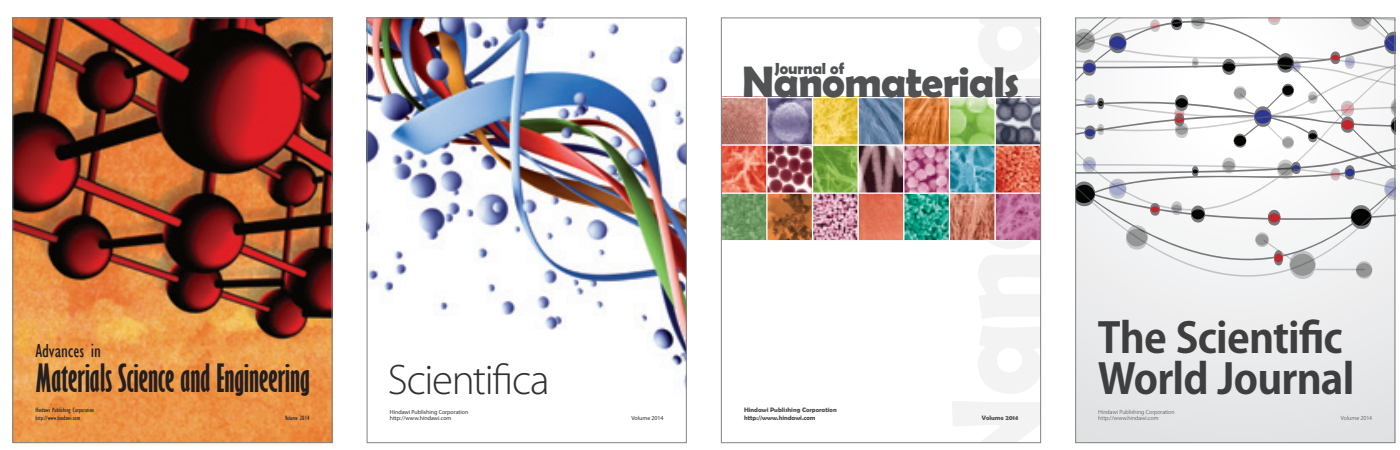

\section{The Scientific World Journal}
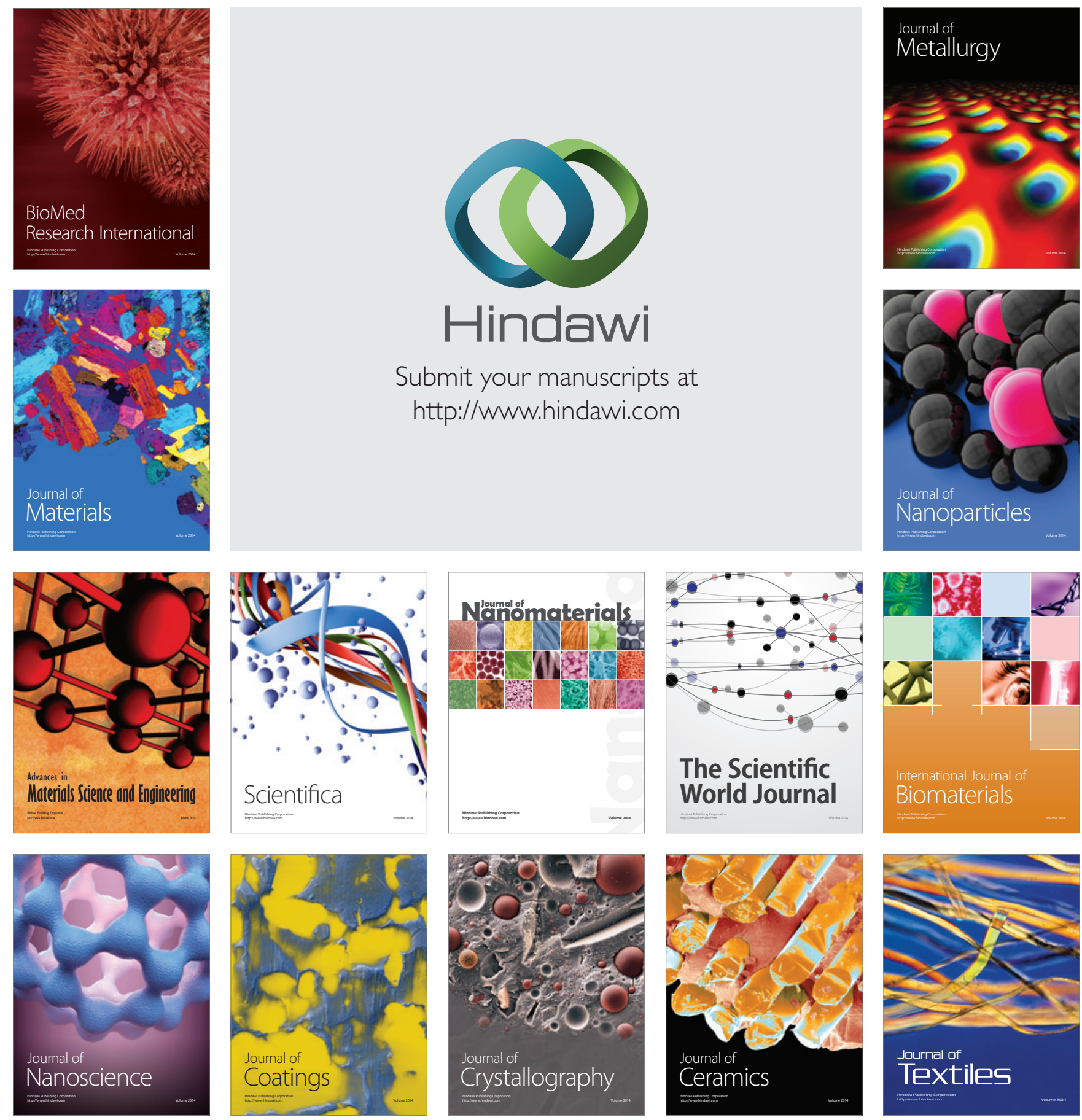